\title{
Propofol activates AMPK to inhibit the growth of HepG2 cells in vitro and hepatocarcinogenesis in xenograft mouse tumor models by inducing autophagy
}

\author{
Yixiong Wang ${ }^{1}$, Baozhu $\mathrm{Xu}^{2}$, Jianying Zhou ${ }^{1}$, Xianyan $\mathrm{Wu}^{1}$ \\ ${ }^{1}$ Department of Anesthesiology, The Quanzhou First Affiliated Hospital of Fujian Medical University, Quanzhou, China; ${ }^{2}$ Department of Radiology, \\ The Second Affiliated Hospital of Fujian Medical University, Quanzhou, China \\ Contributions: (I) Conception and design: All authors; (II) Administrative support: All authors; (III) Provision of study materials or patients: All \\ authors; (IV) Collection and assembly of data: All authors; (V) Data analysis and interpretation: All authors; (VI) Manuscript writing: All authors; (VII) \\ Final approval of manuscript: All authors. \\ Correspondence to: Baozhu Xu. Department of Radiology, The Second Affiliated Hospital of Fujian Medical University, Quanzhou, China. \\ Email: feingjbqzdod@qq.com.
}

Background: Hepatocellular carcinoma (HCC) is a fatal malignant tumor with a poor prognosis, and is the third leading cause of cancer-related deaths worldwide. This study aimed to investigate the anti-tumor effect of propofol on the proliferation, apoptosis, and cell cycle of HCC by regulating adenosine monophosphateactivated protein kinase (AMPK) in vivo and in vitro.

Methods: The cell counting Kit-8 (CCK-8) assay was employed to screen the effect of propofol on HepG2 cell viability at various concentrations $(0.3,0.6,1.2,2.5,5,10,20,40,80$ and $160 \mu \mathrm{M})$. We selected propofol at concentrations of 5, 10 and $20 \mu \mathrm{M}$ for subsequent experiments. Flow cytometry was used to examine the apoptosis and cell cycle of HCC. Quantitative real-time reverse transcription-polymerase chain reaction (qRT-PCR) was applied to measure the messenger ribonucleic acid (mRNA) expression levels of proliferating cell nuclear antigen (PCNA) and survivin. Western blotting was applied to measure the protein expression levels of PCNA, survivin, cleaved caspase-3, cleaved caspase-9, p27 (Kip1), and cyclin A. The effects of propofol were evaluated by establishing a xenograft tumor model.

Results: After treatment with propofol, the mRNA expression levels of PCNA and survivin were decreased compared with the $0 \mu \mathrm{M}$ propofol (control) group. The colony formation assay showed that the colony formation rate was obviously down-regulated. Flow cytometry demonstrated that HepG2 cell apoptosis was increased. G0/G1 was enhanced compared with the control group, while G2/M was restrained. The levels of cleaved caspase-3, cleaved caspase-9, p27, phospho-AMP-activated protein kinase $\alpha 1$ (p-AMPK $\alpha 1$ ), phospho-mammalian target of rapamycin (p-mTOR), and phospho-Unc-51 like autophagy activating kinase 1 (p-ULK1) were notably elevated, while the levels of cyclin A were suppressed. The xenograft tumor volume declined in vivo compared with the HepG2 xenograft group. The expression levels of cell proliferation markers (PCNA) were significantly down-regulated markedly, while the expression levels of cell cycle markers (p27) were notablyup-regulated. Terminal deoxynucleotidyl transferase dUTP nick end labelling (TUNEL) staining showed that cell apoptosis was increased. The levels of p-AMPK 1 were also up-regulated.

Conclusions: Propofol inhibits the proliferation, apoptosis, and cell cycle of HCC by regulating AMPK in vivo and in vitro.

Keywords: Hepatocellular carcinoma (HCC); propofol; proliferation; apoptosis; cell cycle

Submitted Sep 18, 2020. Accepted for publication Nov 26, 2020.

doi: 10.21037/jgo-20-472

View this article at: http://dx.doi.org/10.21037/jgo-20-472 


\section{Introduction}

Hepatocellular carcinoma (HCC) is a fatal malignant tumor with a poor prognosis, and is the third leading cause of cancer-related deaths worldwide (1). With the deterioration of China's ageing population, the burden caused by HCC mortality may yet be a huge challenge for China in the future (2). In China, HCC is primarily caused by viral factors. The current priorities strategy for reducing HCC is to promote hepatitis B vaccination, prevent the spread of hepatitis $C$, and reduce alcohol consumption (3). Surgical resection and liver transplantation are still the standard therapeutic options for the treatment of HCC (4). For surgical resection, the choice of anesthetic drugs plays an important role in the subsequent treatment of HCC. Previous studies have shown that anesthetic drugs strongly accelerate the postoperative progression of spontaneous lung metastases from 3LL Lewis lung carcinoma andB16 melanoma (5). However, emerging research has increasingly shown that anesthetic drugs can reduce the metastasis-promoting activities induced by cancer surgery as a result of immune responses to physiological and anatomical trauma (6). In addition, bupivacaine exhibits direct anti-cancer activity, and its migration-inhibiting effect on gastric cancer is superior to its growth- and survival-inhibiting effect (7).

At present, propofol (2,6-diisopropylphenol), a general sedative and hypnotic reagent, is the most important intravenous general anesthetic in clinical use (8). Propofol anesthesia has anti-tumor protection, inhibits cyclooxygenase 2 and prostaglandin E2 in cancer cells, and stimulates an immune response (9). Propofol inhibits the glycolysis of human umbilical vein endothelial cells and then reduces the adhesion of tumors to endothelial cells (10). Propofol also promotes cisplatin-induced cell apoptosis of cervical cancer cells via the epidermal growth factor receptor (EGFR)/Janus kinase 2 (JAK2)/signal transducer and activator of transcription 3 (STAT3) pathway (11). Furthermore, it inhibits PC12 cell proliferation, prevents cell migration and invasion, activates cell apoptosis, and induces G1/ $S$ phase arrest in malignant pheochromocytoma (12). Notably, propofol sedation represents a valid alternative to general anesthesia for percutaneous liver tumor ablation. Propofol mitigates the periprocedural perception of anxiety and pain, reduces the number of repeat procedures, decrease patient movements, and results in better control of breathing (13). Propofol inhibits HCC growth and invasion through the high mobility group A2 (HMGA2)-mediated Wnt/3-catenin pathway (14). Propofol suppresses HCC by inhibiting neuroepithelial cell transforming gene 1 (NET1) through downregulating extracellular signal-regulated kinase (ERK)/ vascular endothelial growth factor (VEGF) signaling pathway (15). Based on the aforementioned research, this study aims to investigate the use of propofol in HCC.

In eukaryotic cells adenosine monophosphate-activated protein kinase (AMPK) is a key regulator of energy balance that is expressed ubiquitously (16). AMPK is closely related to the tumor suppressor function of liver kinase B1 (LKB1) and tumor protein $\mathrm{p} 53$, thereby regulating the activity of cell survival signals such as mammalian target of rapamycin (mTOR) and protein kinase B (Akt), leading to inhibition of cell growth and cell cycle arrest (17). It has been reported that the overexpression of large tumor suppressor gene 2 (LATS2) can attenuate the treatment resistance of HCC HepG2 cells to sorafenib-mediated death by suppressing the AMPK-mitofusin 2 (Mfn2) signaling pathway (18). Inhibition of glycogen synthase kinase-3 beta (GSK$3 \beta$ ) activity inhibits the HCC malignant phenotype by suppressing glycolysis by enhancing AMPK/mTOR signaling (19). Importantly, AMPK may serve as a novel target to overcome chemoresistance in HCC (20). Based on the foregoing research, this study aims to explore the effect of propofol on the AMPK signaling pathway in HCC. We present the following article in accordance with the ARRIVE reporting checklist (available at http://dx.doi. org/10.21037/jgo-20-472).

\section{Methods}

\section{Cell culture}

The HepG2 cells used in this study were purchased from Chinese Type Culture Collection. The cells were cultured in Roswell Park Memorial Institute (RPMI) 1640 medium (Gibco, USA) [containing 10\% fetal bovine serum (FBS), $1 \times 10^{5} \mathrm{U} / \mathrm{L}$ penicillin, and $1 \times 10^{5} \mathrm{U} / \mathrm{L}$ streptomycin], and then incubated at $37^{\circ} \mathrm{C}$ with $5 \%$ carbon dioxide $\left(\mathrm{CO}_{2}\right)$. The RPMI 1640 medium, FBS, penicillin-streptomycin, and trypsin were purchased from Gibco, USA. The medium was changed every 2 days. This experiment used cells in the logarithmic growth phase.

\section{Quantitative real-time reverse transcription-polymerase chain reaction ( $q R T-P C R)$}

Total ribonucleic acid (RNA) in HepG2 cells was isolated 
using a TRIzol reagent kit (Invitrogen, Beijing, China) according to the manufacturer's protocol. Total RNA concentration was measured using Gene Quant ProRNA/ DNA Calculator (Amersham Pharmacia Biotec, UK). The PrimeScript RT Reagent Kit (TakaRa, Dalian, China) was used to perform reverse transcription. The 2 SYBR Premix Ex Taq $^{\mathrm{TM}}$ II (TakaRa, Dalian, China) were employed to assemble the qRT-PCR reaction system, and the reaction system was carried out in the Bio-Rad CFX-96 (Bio-Rad, CA, USA). Glyceraldehyde 3-phosphate dehydrogenase (GAPDH) was used for normalizing. QRT-PCR data were analyzed using the $2^{-\Delta \Delta \mathrm{Ct}}$ method to calculate the relative expression levels of messenger ribonucleic acid (mRNA). The primers used: GAPDH (Forward: 5'-GAA GGT GAA GGT CGG AGT C-3', Reverse: 5'-GAA GAT GGT GAT GGG ATT C-3'), PCNA (Forward: 5'-AAC CTC ACC AGT ATG TCC AA-3', Reverse: 5'-ACT TTC TCC TGG TTT GGT G-3'), Survivin (Forward: 5'-ACC AGA CCC TCA TGG CTA CC-3', Reverse 5'-CCA TAG CCC AGA AGC CTC ATT-3'), p27(Forward 5'-TCT ACT GCG TGG CTT GTC AG-3', Reverse: 5'-CTG TAT TTG GAG GCA CAG CA-3') and Cyclin A (Forward: 5'CAG AGG CCG AAG ACG AGA C-3', Reverse: 5'-TCA GCT GGC TTC TTC TGA GC-3').

\section{Cell counting Kit-8 (CCK-8) viability assays}

HepG2 cell viability was evaluated using a CCK-8 (Dojindo Molecular Technologies Inc., Kumamoto, Japan) according to the manufacturer's instructions. Finally, the absorbance was read using a microplate reader at $450 \mathrm{~nm}$ (Bio-Rad, California, USA).

\section{Flow cytometry analysis}

HepG2 cell apoptosis was assessed using an Annexin V Staining Kit (BD Biosciences, San Diego, CA, USA). Briefly, the cells were harvested, washed with cold PBS and resuspended in binding buffer, and then incubated with Annexin V and PI for $15 \mathrm{~min}$ at room temperature. Sorting was performed, and the cells were analyzed using BD FACSCanto II.

\section{Western blot assay}

Proteins were isolated from HepG2 cells using a radioimmunoprecipitation assay (RIPA) lysis buffer
(Beyotime Institute of Biotechnology, Shanghai, China) according to the manufacturer's protocol. A bicinchoninic acid (BCA) Protein Assay Kit (Beyotime Institute of Biotechnology, Shanghai, China) was employed to detect the protein concentrations, and the proteins were separated using $10 \%$ dodecyl sulfate-polyacrylamide gel electrophoresis (SDS-PAGE). Subsequently, the proteins were transferred onto polyvinylidene difluoride (PVDF) membranes (Merck Millipore, Germany) by electroblotting. Next, the membranes were blocked with $5 \%$ skim milk in Tris Buffered Saline Tween (TBST, Shanghai Biyuntian Biological Co., Ltd., Shanghai) for $1 \mathrm{~h}$, and incubated overnight at $4{ }^{\circ} \mathrm{C}$ with primary antibody rabbit anti-cleaved caspase- 3 antibody (1:100, ab2302, abcam, UK), anti-anti-cleaved caspase-9 antibody $(1: 1,000$, ab2324, abcam, UK), anti-p27 (1:5,000, ab32034, abcam, UK), anticyclin A (1:1,000, ab185619, abcam, UK), anti-phosphoAMPK $\alpha 1$ (p-AMPK $\alpha 1,1: 1,000$, ab133448, abcam, UK), anti-phosphor-mammalian target of rapamycin (p-mTOR, 1:1,000, ab109268, abcam, UK), and anti-phosphoUnc-5 1 like autophagy activating kinase 1 (p-ULK1, 1:1,000, ab229909, abcam, UK). The membranes were subsequently incubated with corresponding antibodies conjugated to horseradish peroxidase (HRP) for $1 \mathrm{~h}$ at room temperature. GAPDH was employed as the internal control to standardize sample loading. The band densities were determined and analyzed using an automatic digital gel image analysis system (Bio-Rad CFX-96, CA, USA).

\section{Nude mouse subcutaneous xenograft model}

All animal experiments were approved by the Quanzhou First Hospital Affiliated of Fujian Medical University and were performed according to the National Institutes of Health (NIH) Guide for the Care and Use of Laboratory Animals. Eighteen Bagg Albino (BALB/c) nude mice were purchased from Fujian Medical University (female, 5-6 weeks old, weight $17.44 \pm 4.28 \mathrm{~g}$ ). A xenograft nude model was established in female BALB/c mice. A $0.1 \mathrm{~mL}$ cell suspension containing about $5 \times 10^{7}$ HepG2 cells was subcutaneously injected into the right flank regions. One week later, tumor size (volume $>200 \mathrm{~mm}^{3}$ ) was used to evaluate whether the tumors had successfully formed. Next, propofol or an equivalent volume of saline solution was injected intraperitoneally every 3 days consecutively for 30 days. The mice were randomly divided into two groups: (I) HepG2 xenograft group and (II) propofol $(35 \mathrm{mg} / \mathrm{kg})$ group (12). 


\section{Immunobistochemical staining}

Tumor tissue samples were placed on slides and fixed with $4 \%$ paraformaldehyde (Sigma-Aldrich, Germany) for $10 \mathrm{~min}$. The slides were subsequently blocked with $1 \%$ bovine serum albumin for $1 \mathrm{~h}$, and incubated at $4{ }^{\circ} \mathrm{C}$ for $12 \mathrm{~h}$ with primary antibody rabbit anti-PCNA antibody (1:10,000, ab29, abcam) and anti-P27 (1:50, ab32034, abcam). Next, the slides were incubated with goat anti-rabbit immunoglobulin G ( $\mathrm{IgG}$ ) HRP-conjugated secondary antibodies for $1 \mathrm{~h}$. The nuclei were stained with 4',6-diamidino-2-phenylindole (DAPI; SigmaAldrich, Germany). Fluorescence imaging was analyzed using confocal laser scanning microscopy (Carl Zeiss, Oberkochen, Germany). The integrated option density (IOD) and area of the myocardial cells were evaluated in each tissue section, and were used to obtain the average optical density value $(\mathrm{OD}=\mathrm{IOD} / \mathrm{area})$. Four slices were selected, and four fields of view were selected for each slice.

\section{Terminal-deoxynucleotidyl transferase mediated nick end labeling (TUNEL) staining}

Part of the tumor tissues were taken to make paraffin sections. After routine deparaffinization, the sections were immersed in $0.1 \mathrm{M} \mathrm{pH} 6.0$ citric acid buffer, microwaved for 5 minutes, and washed twice with PBS. They were then added to the TUNEL reaction solution in a wet box at $37^{\circ} \mathrm{C}$ for $1 \mathrm{~h}$ in the dark, and washed with PBS three times. The slides were stained with TUNEL using the in situ cell death detection kit, POD (Roche, Penzberg, Germany). POD was added to the slices in a humid chamber at $37^{\circ} \mathrm{C}$ for $30 \mathrm{~min}$, washed three times with PBS, and dried. 3,3'-Diaminobenzidine (DAB) was added at room temperature for $10 \mathrm{~min}$, counterstained, dehydrated with gradient ethanol, clarified with xylene, and sealed with neutral glue. The sections were observed under an OLYMPUS DX51 fluorescence microscope (Tokyo, Japan), and five fields of view were randomly selected for image analysis using image6.0 (Media Cybernetics, USA). The TUNEL cell apoptosis detection kit (C1091) was purchased from Shanghai Biyuntian Institute of Biotechnology (Shanghai, China).

\section{Statistical analysis}

All experiments were carried out independently at least three times, and all experimental data were expressed as mean \pm standard deviation (SD). The Student's $t$ test and SPSS 22.0 software (SPSS Inc.) were used for statistical analysis between the two groups. $\mathrm{P}<0.05$ was considered statistically significant.

\section{Results}

\section{Propofol inbibited HepG2 cell proliferation}

HepG2 cells were treated with different concentrations of propofol for $24 \mathrm{~h}$. The results showed that HepG2 cell viability was decreased significantly as the concentration of propofol increased (Figure 1A). According to the results of the cell viability experiments, we selected propofol at a concentration of 5,10 , and $20 \mu M$ for subsequent experiments. After treatment with propofol, the mRNA and protein expression levels of PCNA and survivin were decreased compared with the $0 \mu \mathrm{M}$ propofol (control) group (Figure $1 B, C, \mathrm{P}<0.05$ ). These results suggested that propofol could inhibit HepG2 cell proliferation.

\section{Propofol promoted HepG2 cell apoptosis}

After treatment with propofol, flow cytometry showed that HepG2 cell apoptosis was increased compared with the control group (Figure $2 A, \mathrm{P}<0.05$ ). The protein expression levels of cleaved caspase- 3 and cleaved caspase- 9 were detected by western blot, and were notably elevated compared with the control group (Figure $2 B, \mathrm{P}<0.05$ ). These results indicate that propofol could promote HepG2 cell apoptosis.

\section{Propofol suppressed the HepG2cell cycle}

The mRNA expression levels of p27 and cyclin A were detected by qRT-PCR, and were notably elevated compared with the control group, while the levels of cyclin A were suppressed (Figure $3 A, \mathrm{P}<0.05$ ). The protein expression levels of p27 and cyclin A were detected by western blot, and were notably elevated compared with the control group, while the levels of cyclin A were suppressed (Figure $3 B, \mathrm{P}<0.05$ ). These results suggested that propofol could suppress the HepG2 cell cycle.

\section{Propofol activated the AMPK signaling pathway in HepG2 cells}

The protein expression levels of p-AMPKa1, p-mTOR and 
A

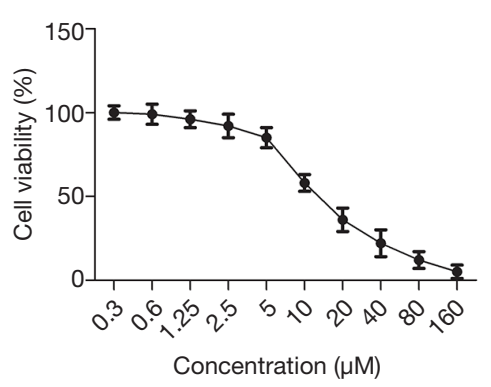

C

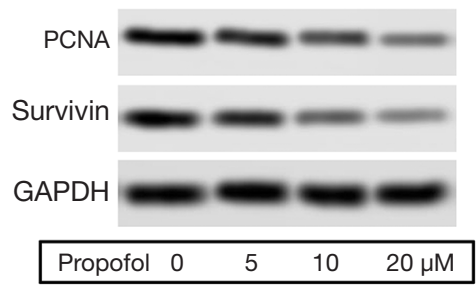

B
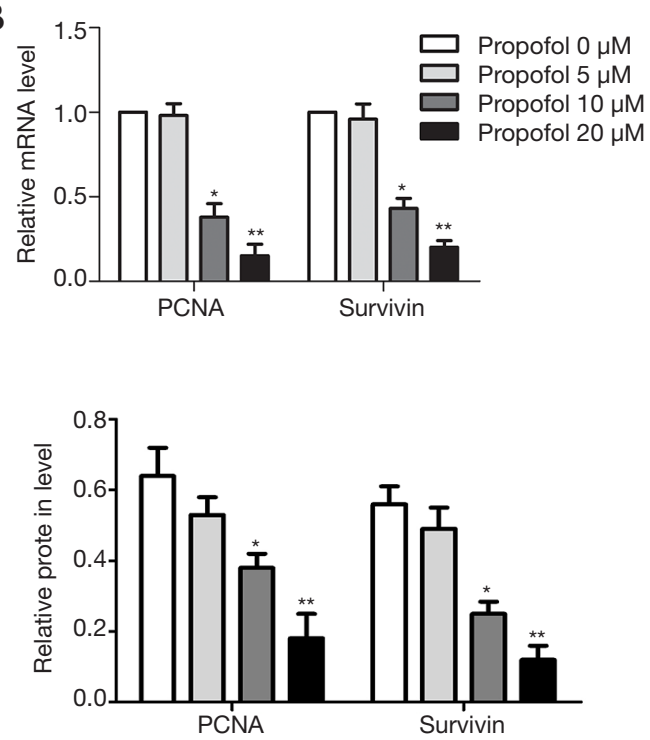

Figure 1 Propofol inhibited HepG2 cell proliferation (A) Effect of propofol (at 0.3, 0.6, 1.2, 2.5, 5, 10, 20, 40, 80 and $160 \mu \mathrm{M}$ ) on HepG2 cell viability were measured using a CCK-8 kit; (B) the mRNA expression levels of PCNA (proliferating cell nuclear antigen) and survivin were detected by qRT-PCR (quantitative real-time reverse transcription-polymerase chain reaction) in the propofol $0 \mu M$ group (control group), propofol $5 \mu \mathrm{M}$ group, propofol $10 \mu \mathrm{M}$ group, and propofol $20 \mu \mathrm{M}$ group; (C) the protein expression levels of PCNA and survivin were detected by western blot. *, $\mathrm{P}<0.05$; **, $\mathrm{P}<0.01$ versus propofol $0 \mu \mathrm{M}$ group.

A
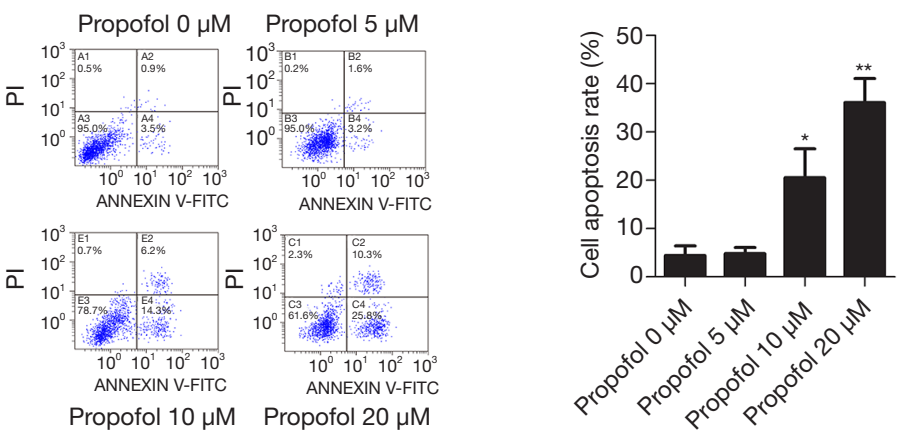

B
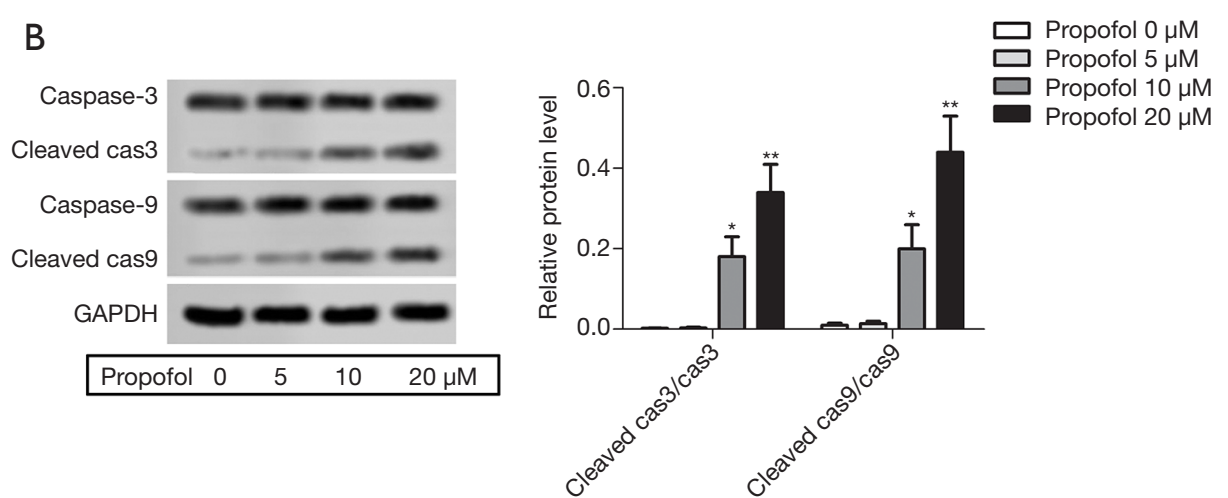

Figure 2 Propofol promoted HepG2 cell apoptosis. (A) Flow cytometry was employed to detect HepG2 cell apoptosis (HepG2 cells apoptosis rates $=$ the second quadrant + the fourth quadrant); (B) the protein expression levels of cleaved caspase- 3 and cleaved caspase- 9 were detected by western blot. Also, the histogram revealed the semi-quantitative analysis of the protein. ${ }^{*}, \mathrm{P}<0.05 ;{ }^{* *}, \mathrm{P}<0.01$ versus propofol $0 \mu \mathrm{M}$ group. 
A
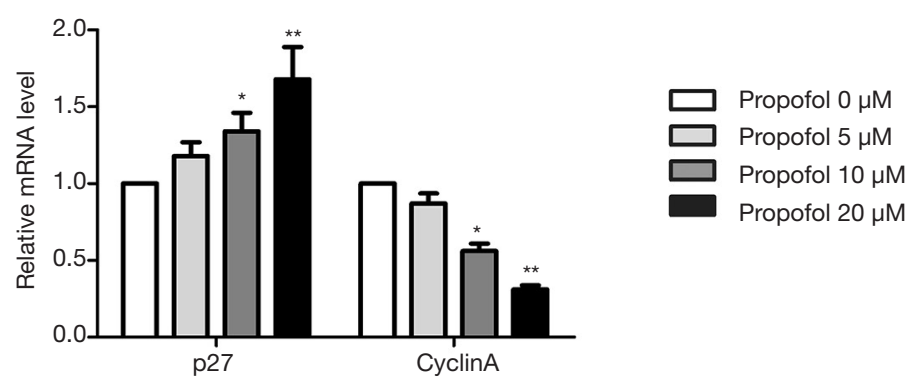

B
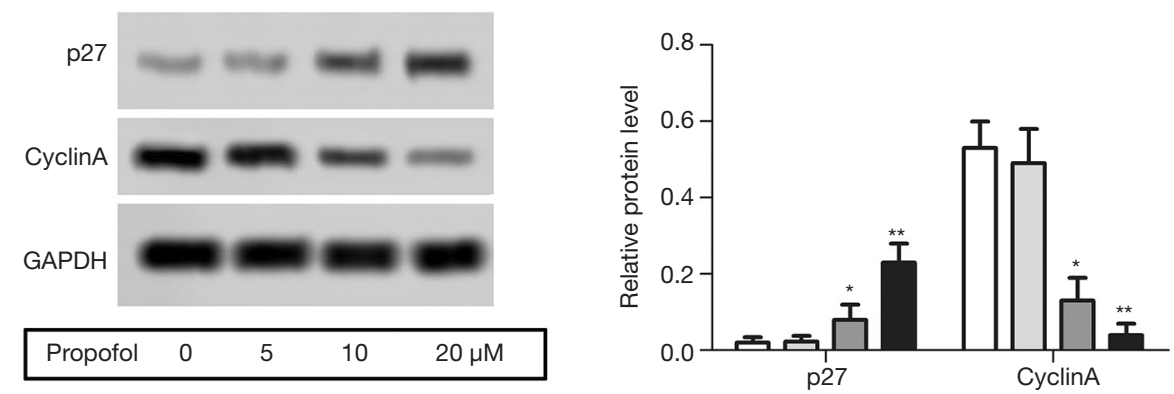

Figure 3 Propofol suppressed the HepG2 cell cycle. (A) The mRNA expression levels of p27 and cyclin A were detected by qRT-PCR (quantitative real-time reverse transcription-polymerase chain reaction); (B) the protein expression levels of p27 and cyclin A were detected by western blot. Also, the histogram revealed the semi-quantitative analysis of the protein. * $\mathrm{P}<0.05 ;{ }^{* *}, \mathrm{P}<0.01$ versus propofol $0 \mu M$ group.

p-ULK1 were detected by western blot. After treatment with propofol, the levels of p-AMPKa1, p-mTOR, and p-ULK1 were notably enhanced compared with the control group (Figure $4 A, B, \mathrm{P}<0.05$ ). These results suggested that propofol could activate the AMPK signaling pathway in HepG2 cells.

\section{The effect of propofol in vivo}

As for the effect of propofol on tumor growth, xenograft tumor weight and volume were declined in vivo compared with the HepG2 xenograft group (Figure $5 A, B, \mathrm{P}<0.05$ ). After treatment with propofol, the expression levels of cell proliferation markers (PCNA) were significantly downregulated compared with the HepG2 xenograft group (Figure 5C, $\mathrm{P}<0.05$ ), and the expression levels of cell cycle markers (p27) were substantially up-regulated compared with the HepG2 xenograft group (Figure 5D, $\mathrm{P}<0.05$ ). Furthermore, TUNEL staining showed that cell apoptosis was increased compared with the HepG2 xenograft group (Figure 5E, $\mathrm{P}<0.05$ ). Moreover, the protein expression levels of p-AMPK $\alpha 1$ were up-regulated compared with the HepG2 xenograft group (Figure $5 F, \mathrm{P}<0.05$ ). These results confirmed that propofol inhibits tumor growth in vivo.

\section{Discussion}

Liver cancer is the most common malignancy of the digestive system, and has a high mortality rate. It is classified into HCC and intrahepatic cholangiocarcinoma (ICC) $(21,22)$. At present, the standard treatment for liver cancer includes surgical resection, cytotoxic chemotherapy, immunotherapy, and oncolytic virus therapy (23). For HCC, surgical resection and liver transplantation are still the basic therapeutic options (4). For surgical resection, the choice of anesthetic drugs plays an important role in the subsequent treatment of HCC. Propofol anaesthesia was associated with better survival in HCC patients who underwent hepatectomy (24). Propofol inhibits cell viability, migration, and invasion, but promotes apoptosis of HepG2 and SMMC-7721 cells. Also, the levels of cleaved caspase-3 and cleaved caspase-9 are up-regulated (25). Previous research has shown that PCNA is a key protein necessary for deoxyribonucleic acid (DNA) replication in proliferating cells (such as cancer cells) (26). Survivin has been extensively investigated using proliferation and apoptosis assays in tumor cells (27). It can inhibit cell death and regulate the cell cycle, especially during the mitotic phase, which all contribute to the progression of cancer. Clinical specimens have demonstrated that the expression of survivin is always 
A

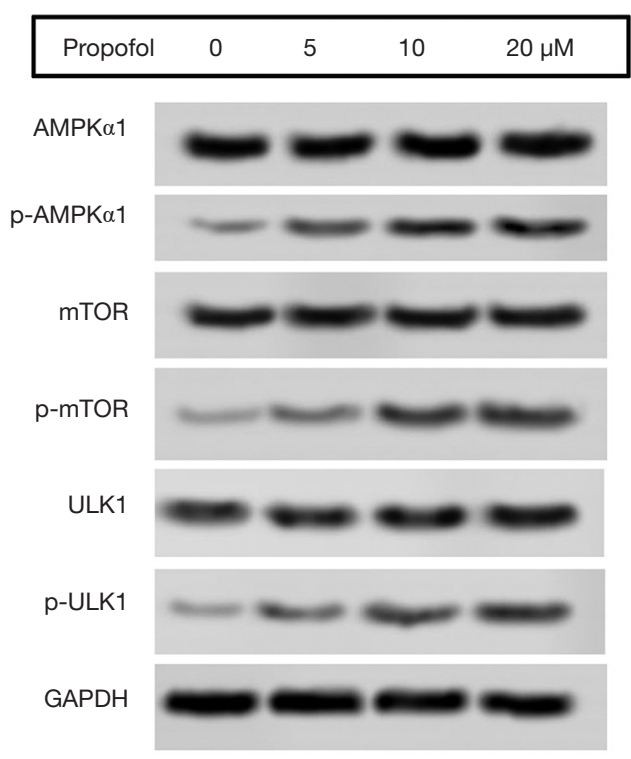

B

$\square$ Propofol $0 \mu \mathrm{M}$

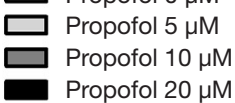

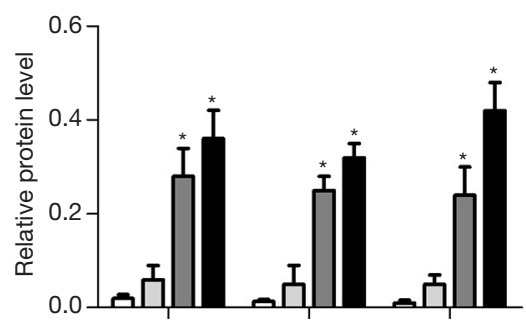

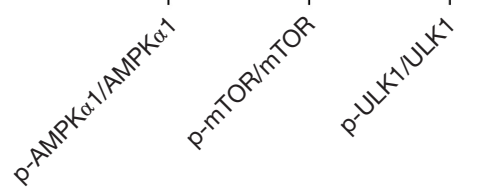

Figure 4 Propofol restrained the AMPK (adenosine monophosphate-activated protein kinase) signaling pathway in HepG2 cells. (A) The protein expression levels of p-AMPK $\alpha 1$ (phospho-AMP-activated protein kinase $\alpha 1$ ), p-mTOR (phospho-mammalian target of rapamycin), and p-ULK1 [phospho-Unc-51 like autophagy activating kinase 1 (p-ULK1)] were detected by western blot; (B) the histogram revealed the semi-quantitative analysis of the protein. *, $\mathrm{P}<0.05$, versus propofol $0 \mu \mathrm{M}$ group.

up-regulated in human cancers and is associated with chemotherapy or radiotherapy resistance, as well as poor prognosis (28).

In our research, the mRNA expression levels of PCNA and survivin were decreased after treatment with propofol. The colony formation assay demonstrated that the colony formation rate was obviously down-regulated after treatment with propofol. Also, flow cytometry exhibited that HepG2 cell apoptosis was increased after treatment with propofol. The levels of cleaved caspase- 3 and cleaved caspase-9 were notably elevated after treatment with propofol. Propofol mediates the transfer of micro RNA (miR)-142-3p from macrophages to cancer cells through microcapsules, exerting anti-HCC effects (29). Propofol also decreases HCC cell invasiveness by enhancing the expression of miR-199a and inhibiting expression of matrix metallopeptidase 9 (MMP-9) (30). These results indicate that propofol could inhibit HepG2 cell proliferation and promote HepG2 cell apoptosis.

Cell cycle progression is closely regulated via a complex network of regulatory molecules. The cell cycle refers to a series of events that occur during cell division that are precisely controlled to adjust cell proliferation, apoptosis, and other events critical to cell survival (31). Loss of normal cell cycle control is a hallmark of cancer (32). P27 not only regulates the cell cycle but also suppresses cell proliferation by impacting the formation of the cell cycledependent complex cell division protein kinase 6 (CDK6)/ cyclin D1 (CCND1) (33). When located in the cytoplasm, p27acquires oncogenic roles, and its levels in non-small cell lung carcinoma patients appear to be a powerful prognostic marker (34). The deletion of p27 occurs in the early stage of carcinogenesis, and is related to the aggressiveness and metastasis of liver cancer. At the same time, it is also a prognostic marker (35). CDK and cyclin are core factors that endogenously regulate the cell cycle (36). Based on the interaction between $\mathrm{p} 27$ and cyclin/CDK, the development of specific cyclin E/cyclin A-CDK2 inhibitors inhibit cell cycle progression, thereby preventing tumor growth and survival (37). In our research, after treatment with propofol, the mRNA and protein expression levels of p27 were notably elevated, while the levels of cyclin A were suppressed. These results suggested that propofol could suppress the HepG2 cell cycle.

AMPK directly promotes autophagy by phosphorylating the autophagy-related proteins in mTOR, Unc-51 like autophagy activating kinase 1 (ULK1), and the phosphatidylinositol 3-kinase catalytic subunit type 3 
A

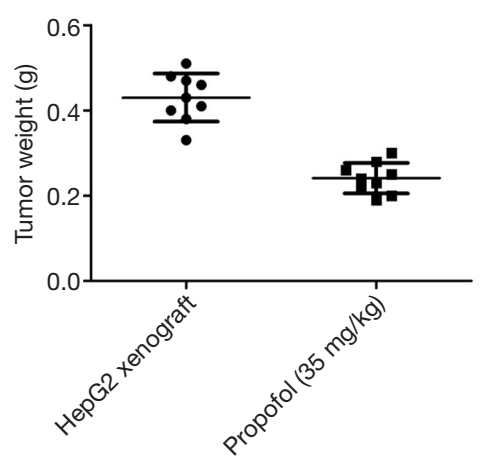

C

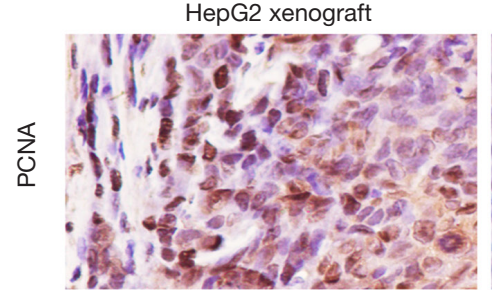

D

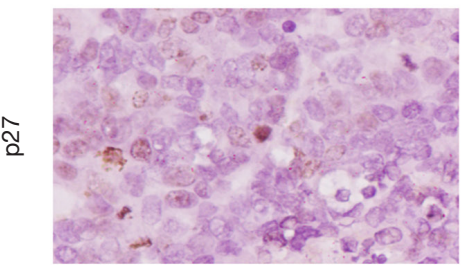

E

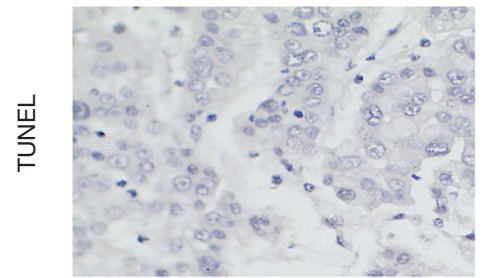

B

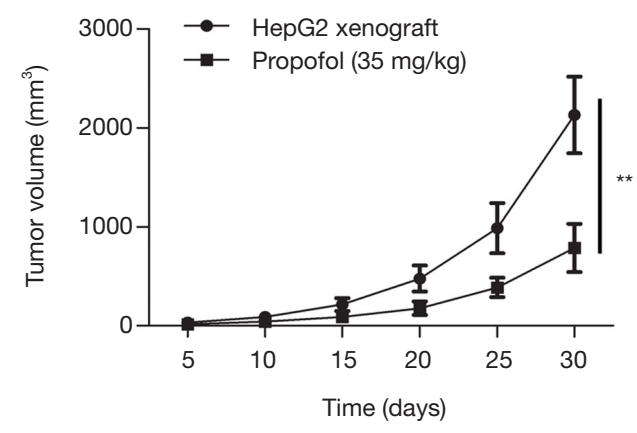

$\mathrm{F}$

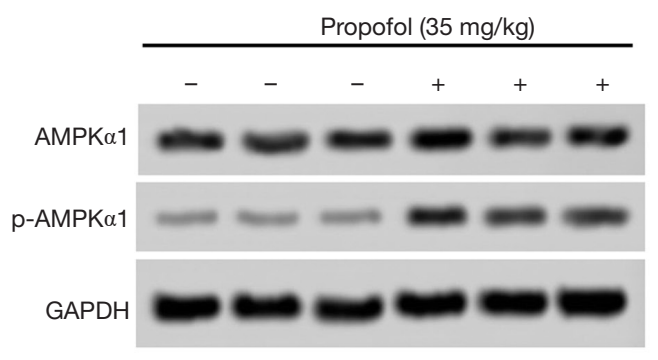

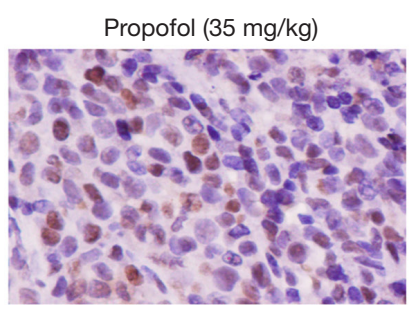
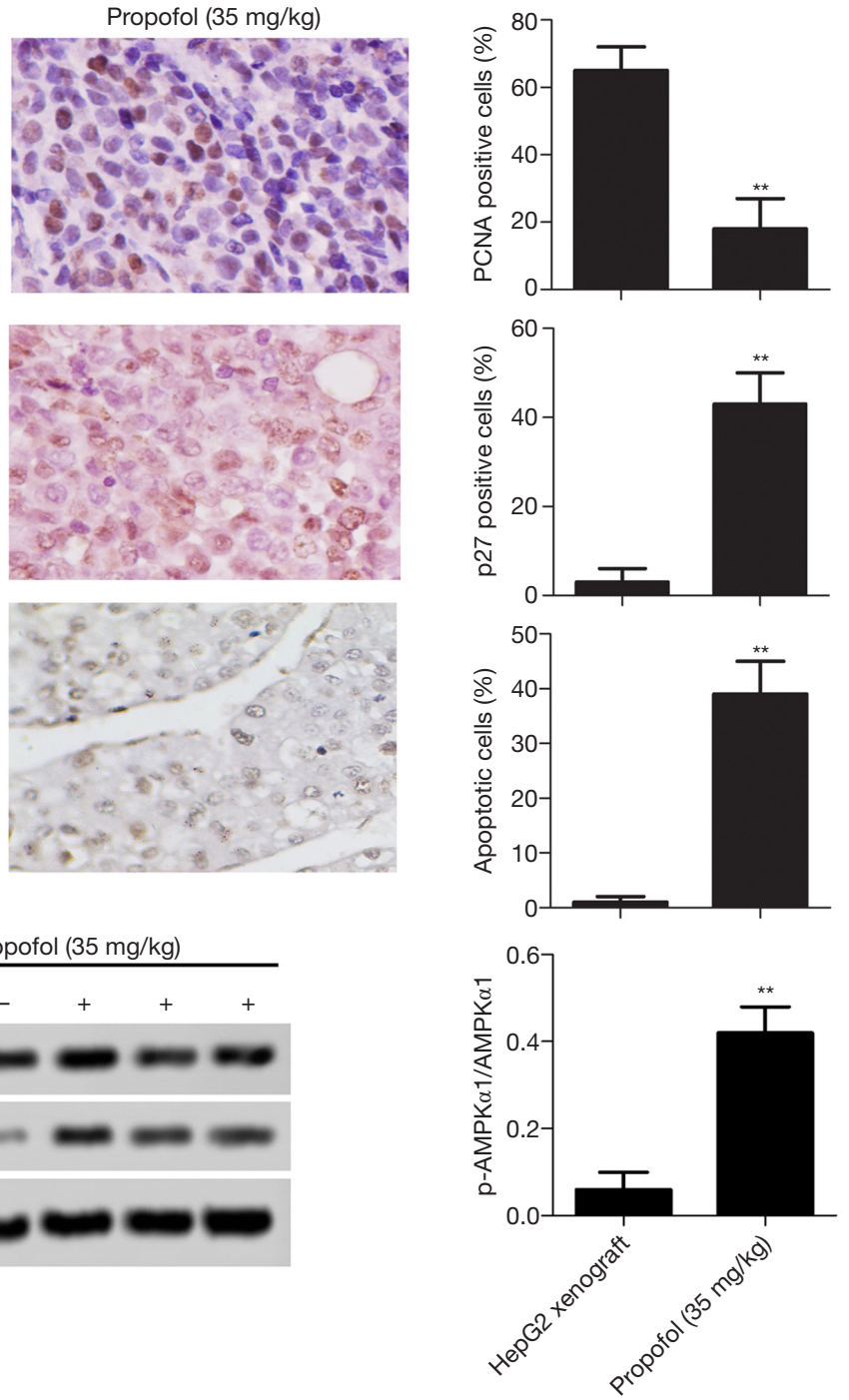

Figure 5 The effect of propofol in vivo. (A,B) The tumor weight and volume was determined; (C) the expression of PCNA (proliferating cell nuclear antigen) was measured by immunohistochemistry analysis (magnification, $\times 400$ ); (D) the expression of p27 was measured by immunohistochemistry analysis (magnification, $\times 400$ ); (E) cell apoptosis was detected by TUNEL staining (magnification, $\times 400$ ); (F) the protein expression levels of p-AMPKa1 (phospho-AMP-activated protein kinase $\alpha 1$ ) were detected by western blot. Also, the histogram revealed the semi-quantitative analysis of the protein. ${ }^{* *}, \mathrm{P}<0.01$ versus HepG2 xenograft group. 
(PIK3C3/VPS34) complex, or indirectly promotes autophagy by regulating the expression of autophagyrelated genes downstream of transcription factors [such as forkhead box O-3 (FoxO3), transcription factor EB (TFEB), and Bromodomain-containing protein 4 (BRD4)] (38). MTOR is a protein kinase that regulates cell growth, survival, metabolism, and immunity. Activation of mTOR promotes tumor growth and metastasis (39). Metformin can induce the levels of DEP domain-containing mTORinteracting protein (DEPTOR), which intensifies binding to $\mathrm{mTOR}$ and exerts a suppressing effect on mTOR signaling in HCC (40). Previous research has shown that propofol suppresses the proliferation, differentiation, and migration of neural stem cells (NSCs), and these functions are partially mediated by the calmodulin-dependent protein kinase (CaMk) II/phosphorylation of serine at amino acid position 485 (pS485)/AMPK/activating transcription factor 5 (ATF5) signaling pathway (41). Importantly, the AMPK/ mTOR signaling pathway has been found to be activated in propofol-induced autophagosome accumulation in HeLa cells (42). Medium- and long-chain triglyceride (MCT/LCT) propofol not only significantly promotes the phosphorylation of AMPK and acetyl coenzyme A carboxylase (ACC), but also reverses the free fatty acid (FFA)-induced decreased phosphorylation of AMPK and ACC (43). In our research, the levels of p-AMPK $\alpha 1$, p-mTOR, and p-ULK1 were notably restrained following treatment with propofol compared with the $0 \mu \mathrm{M}$ propofol group. These results indicated that propofol could restrain the AMPK signaling pathway in HepG2 cells.

In conclusion, these results demonstrate that propofol inhibited the proliferation and cell cycle of HepG2 cells, and promoted HepG2 cell apoptosis. Treatment with propofol also activated the AMPK signaling pathway in HepG2 cells. In addition, propofol activated the AMPK signaling pathway in vivo. For patients with HCC, propofol is a more advantageous option during surgery.

\section{Acknowledgments}

Funding: None.

\section{Footnote}

Reporting Checklist: The authors have completed the ARRIVE reporting checklist. Available at http://dx.doi. org/10.21037/jgo-20-472
Data Sharing Statement: Available at http://dx.doi. org/10.21037/jgo-20-472

Conflicts of Interest: All authors have completed the ICMJE uniform disclosure form (available at http://dx.doi. org/10.21037/jgo-20-472). The authors have no conflicts of interest to declare.

Ethical Statement: The authors are accountable for all aspects of the work in ensuring that questions related to the accuracy or integrity of any part of the work are appropriately investigated and resolved. All animal experiments were approved by the Quanzhou First Hospital Affiliated of Fujian Medical University and were performed according to the National Institutes of Health (NIH) Guide for the Care and Use of Laboratory Animals.

Open Access Statement: This is an Open Access article distributed in accordance with the Creative Commons Attribution-NonCommercial-NoDerivs 4.0 International License (CC BY-NC-ND 4.0), which permits the noncommercial replication and distribution of the article with the strict proviso that no changes or edits are made and the original work is properly cited (including links to both the formal publication through the relevant DOI and the license). See: https://creativecommons.org/licenses/by-nc-nd/4.0/.

\section{References}

1. Shiani A, Narayanan S, Pena L, et al. The Role of Diagnosis and Treatment of Underlying Liver Disease for the Prognosis of Primary Liver Cancer. Cancer Control 2017;24:1073274817729240.

2. Sun Y, Wang Y, Li M, et al. Long-term trends of liver cancer mortality by gender in urban and rural areas in China: an age-period-cohort analysis. BMJ Open 2018;8:e020490.

3. Pocha C, Xie C. Hepatocellular carcinoma in alcoholic and non-alcoholic fatty liver disease-one of a kind or two different enemies? Transl Gastroenterol Hepatol 2019;4:72.

4. Chawla A, Ferrone C. Hepatocellular carcinoma surgical therapy: perspectives on the current limits to resection. Chin Clin Oncol 2018;7:48.

5. Shapiro J, Jersky J, Katzav S, et al. Anesthetic drugs accelerate the progression of postoperative metastases of mouse tumors. J Clin Invest 1981;68:678-85. 
6. Vahabi S, Eatemadi A. Effects of anesthetic and analgesic techniques on cancer metastasis. Biomed Pharmacother 2017;87:1-7.

7. Dan J, Gong X, Li D, et al. Inhibition of gastric cancer by local anesthetic bupivacaine through multiple mechanisms independent of sodium channel blockade. Biomed Pharmacother 2018;103:823-8.

8. Yip GM, Chen ZW, Edge CJ, et al. A propofol binding site on mammalian GABAA receptors identified by photolabeling. Nat Chem Biol 2013;9:715-20.

9. Fodale V, D'Arrigo MG, Triolo S, et al. Anesthetic techniques and cancer recurrence after surgery. ScientificWorldJournal 2014;2014:328513.

10. Qi J, Wu Q, Zhu X, et al. Propofol attenuates the adhesion of tumor and endothelial cells through inhibiting glycolysis in human umbilical vein endothelial cells. Acta Biochim Biophys Sin (Shanghai) 2019;51:1114-22.

11. Li H, Lu Y, Pang Y, et al. Propofol enhances the cisplatininduced apoptosis on cervical cancer cells via EGFR/JAK2/ STAT3 pathway. Biomed Pharmacother 2017;86:324-33.

12. Wang H, Zhang S, Zhang A, et al. Propofol Prevents the Progression of Malignant Pheochromocytoma In Vitro and In Vivo. DNA Cell Biol 2018;37:308-15.

13. Puijk RS, Ziedses des Plantes V, Nieuwenhuizen S, et al. Propofol Compared to Midazolam Sedation and to General Anesthesia for Percutaneous Microwave Ablation in Patients with Hepatic Malignancies: A Single-Center Comparative Analysis of Three Historical Cohorts. Cardiovasc Intervent Radiol 2019;42:1597-608.

14. Chung J, Zhang X, Collins B, et al. High mobility group A2 (HMGA2) deficiency in pigs leads to dwarfism, abnormal fetal resource allocation, and cryptorchidism. Proc Natl Acad Sci U S A 2018;115:5420-5.

15. Fei G, Cao M, Ge C, et al. Propofol suppresses hepatocellular carcinoma by inhibiting NET1 through downregulating ERK/VEGF signaling pathway. Sci Rep 2020;10:11208.

16. Carling D. AMPK signalling in health and disease. Curr Opin Cell Biol 2017;45:31-7.

17. Wang Z, Wang N, Liu P, et al. AMPK and Cancer. Exp Suppl 2016;107:203-26.

18. Song J, Zhao W, Lu C, et al. LATS2 overexpression attenuates the therapeutic resistance of liver cancer HepG2 cells to sorafenib-mediated death via inhibiting the AMPK-Mfn2 signaling pathway. Cancer Cell Int 2019;19:60.

19. Fang G, Zhang P, Liu J, et al. Inhibition of GSK$3 \beta$ activity suppresses HCC malignant phenotype by inhibiting glycolysis via activating AMPK/mTOR signaling. Cancer Lett 2019;463:11-26.

20. Bort A, Sánchez BG, Mateos-Gómez PA, et al. Targeting AMP-activated kinase impacts hepatocellular cancer stem cells induced by long-term treatment with sorafenib. Mol Oncol 2019;13:1311-31.

21. Yamashita T, Kaneko S. Liver Cancer. Rinsho Byori 2016;64:787-96.

22. Orcutt ST, Anaya DA. Liver Resection and Surgical Strategies for Management of Primary Liver Cancer. Cancer Control 2018;25:1073274817744621.

23. Liu CY, Chen KF, Chen PJ. Treatment of Liver Cancer. Cold Spring Harb Perspect Med 2015;5:a021535.

24. Lai HC, Lee MS, Lin C, et al. Propofol-based total intravenous anaesthesia is associated with better survival than desflurane anaesthesia in hepatectomy for hepatocellular carcinoma: a retrospective cohort study. $\mathrm{Br}$ J Anaesth 2019;123:151-60.

25. Liu SQ, Zhang JL, Li ZW, et al. Propofol Inhibits Proliferation, Migration, Invasion and Promotes Apoptosis Through Down-Regulating miR-374a in Hepatocarcinoma Cell Lines. Cell Physiol Biochem 2018;49:2099-110.

26. Zhao H, Lo YH, Ma L, et al. Targeting tyrosine phosphorylation of PCNA inhibits prostate cancer growth. Mol Cancer Ther 2011;10:29-36.

27. Lin Y, Yue B, Xiang H, et al. Survivin is expressed in degenerated nucleus pulposus cells and is involved in proliferation and the prevention of apoptosis in vitro. Mol Med Rep 2016;13:1026-32.

28. Yamamoto H, Ngan CY, Monden M. Cancer cells survive with survivin. Cancer Sci 2008;99:1709-14.

29. Zhang J, Shan WF, Jin TT, et al. Propofol exerts antihepatocellular carcinoma by microvesicle-mediated transfer of miR-142-3p from macrophage to cancer cells. J Transl Med 2014;12:279.

30. Zhang J, Zhang D, Wu GQ, et al. Propofol inhibits the adhesion of hepatocellular carcinoma cells by upregulating microRNA-199a and downregulating MMP-9 expression. Hepatobiliary Pancreat Dis Int 2013;12:305-9.

31. Hartwell LH, Weinert TA. Checkpoints: controls that ensure the order of cell cycle events. Science 1989;246:629-34.

32. Aarts M, Linardopoulos S, Turner NC. Tumour selective targeting of cell cycle kinases for cancer treatment. Curr Opin Pharmacol 2013;13:529-35.

33. Li N, Zeng J, Sun F, et al. p27 inhibits CDK6/ CCND1 complex formation resulting in cell cycle arrest and inhibition of cell proliferation. Cell Cycle 
2018;17:2335-48.

34. Calvayrac O, Nowosad A, Cabantous S, et al. Cytoplasmic p27Kip1 promotes tumorigenesis via suppression of RhoB activity. J Pathol 2019;247:60-71.

35. Chetty R. p27 Protein and cancers of the gastrointestinal tract and liver: an overview. J Clin Gastroenterol 2003;37:23-7.

36. Tan H, Gao S, Zhuang Y, et al. R-Phycoerythrin Induces SGC-7901 Apoptosis by Arresting Cell Cycle at S Phase. Mar Drugs 2016;14:166.

37. Dai L, Liu Y, Liu J, et al. A novel cyclin E/cyclin A-CDK inhibitor targets p27(Kip1) degradation, cell cycle progression and cell survival: implications in cancer therapy. Cancer Lett 2013;333:103-12.

38. Li Y, Chen Y. AMPK and Autophagy. Adv Exp Med Biol 2019;1206:85-108.

39. Hua H, Kong Q, Zhang H, et al. Targeting mTOR for cancer therapy. J Hematol Oncol 2019;12:71.

40. Obara A, Fujita Y, Abudukadier A, et al. DEPTOR-related
mTOR suppression is involved in metformin's anti-cancer action in human liver cancer cells. Biochem Biophys Res Commun 2015;460:1047-52.

41. Liang C, Du F, Wang J, et al. Propofol Regulates Neural Stem Cell Proliferation and Differentiation via Calmodulin-Dependent Protein Kinase II/AMPK/ATF5 Signaling Axis. Anesth Analg 2019;129:608-17.

42. Chen X, Li K, Zhao G. Propofol Inhibits HeLa Cells by Impairing Autophagic Flux via AMP-Activated Protein Kinase (AMPK) Activation and Endoplasmic Reticulum Stress Regulated by Calcium. Med Sci Monit 2018;24:2339-49.

43. Wang LY, Wu J, Gao YF, et al. Medium- and longchain triglyceride propofol reduces the activity of acetylcoenzyme A carboxylase in hepatic lipid metabolism in HepG2 and Huh7 cells. Korean J Physiol Pharmacol 2020;24:19-26.

(English Language Editor: A. Kassem)

Cite this article as: Wang Y, Xu B, Zhou J, Wu X. Propofol activates AMPK to inhibit the growth of HepG2 cells in vitro and hepatocarcinogenesis in xenograft mouse tumor models by inducing autophagy. J Gastrointest Oncol 2020;11(6):1322-1332. doi: 10.21037/jgo-20-472 